\title{
Socialisation, sociologie des champs et psychanalyse. Jusqu'où pousser l'unité des sciences sociales?
}

Marc Joly

Grâce à sa précocité, à sa productivité jamais démentie et à l'originalité de son projet de refondation théorique de la sociologie, Bernard Lahire, né en 1963, s'est acquis une place de premier plan dans le paysage des sciences humaines et sociales françaises. À bien des égards, on pourrait dire que toute son entreprise a consisté à prendre au sérieux l'idée suivante, énoncée à la fin des années 1920, avec un brin de provocation, par un jeune philosophe qui plaidait pour une collaboration étroite entre la psychologie et la sociologie : «Le meilleur point de vue pour étudier les phénomènes sociaux, c'est l'individu» (Essertier 1927: 23). Cela traduit un intérêt de connaissance que Lahire, en ce qui le concerne, invite à interpréter par rapport à sa trajectoire personnelle et à la triple nécessité d'ordre scientifique qu'il dit avoir ressenti après sa thèse de doctorat sur l'« échec scolaire » à l'école primaire (1993) : (1) changer d'échelle d'observation et de niveau d'analyse des faits sociaux; (2) faire porter l'éclairage sur les réalités familiales et sur la pluralité des cadres de socialisation traversés par les individus; (3) rendre compte sociologiquement, par là, des «cas improbables » (2012b : 11-20). La publication de Monde pluriel (2012a), qui fait suite à L'Homme pluriel (1998), offre l'opportunité de discuter de plain-pied la portée théorique de ce changement de perspective ${ }^{\mathrm{i}}$.

Dans ce livre, l'ambition de Lahire est de penser tout à la fois le phénomène historique de la différenciation sociale et l'unité théorique des sciences humaines et sociales, alors même que la différenciation des fonctions sociales, sous la forme spécifique particulièrement accusée qu'elle revêt dans le domaine scientifique, représente l'un des principaux obstacles à surmonter pour produire une « image du monde social dans son ensemble » (2012a : 323). Le sociologue se donne 
ainsi pour objectif d'examiner «les travaux et réflexions sociologiques, historiques ou anthropologiques majeurs concernant la question de la différenciation et de l'autonomisation des univers sociaux » (ibid. : 62). Et, à partir de cette lecture critique (il parle d'une «fréquentation lente, rigoureuse et précise des textes » [ibid. : 20], et c'est assurément l'un des points forts de Monde pluried) et des acquis de ses propres recherches sur la constitution des patrimoines de dispositions individuelles dans les sociétés contemporaines, il se propose de renouveler la théorie bourdieusienne de la pratique et d'offrir aux différentes disciplines des sciences humaines et sociales (et aux multiples paradigmes qui se sont développés en leur sein) une base commune de discussion. C'est donc vers une même théorie de la pratique - elle-même inséparable, principiellement, d'un programme de sociologie des singularités individuelles - que les sciences humaines et sociales semblent destinées à converger le plus aisément.

\section{De la différenciation sociale à l'unité des sciences sociales}

Pour Lahire, penser la différenciation sociale revient à penser l'opération même de contextualisation (puisque cerner la dynamique et le fonctionnement des sociétés différenciées implique de toujours relier les pratiques des individus aux contextes dûment spécifiés - du plus large au plus étroit - à travers lesquels ils ont formé leurs dispositions et agissent). Et penser l'opération de contextualisation revient à prendre position épistémologiquement en faveur d'une synthèse du « réalisme » et du «nominalisme » (puisque les contextes sont à la fois des réalités qui doivent être correctement identifiées en tant que telles et des constructions qui dépendent des intérêts de connaissance du chercheur et des problèmes qu'il aspire à résoudre). Tout se tient, ainsi, dans la démonstration du sociologue. Peut-être aurait-il pu reconnaître plus explicitement que la théorie de la pratique qu'il expose est indissociablement une théorie de la différenciation sociale et de la socialisation (ou, pour être plus précis, une théorie de la socialisation dans les 
sociétés différenciées qui est également - ou devrait être - une théorie du psychisme). Une formule suffit en tout cas à la faire comprendre : «Dispositions ou compétences + Contexte = Pratiques ». Une telle formule, par sa généralité et sa simplicité, a pour vocation de servir de point de ralliement autour duquel les sciences humaines et sociales pourraient dialoguer en dépit de leurs différences. D’autant qu'elle a pour corrélat une épistémologie postulant la pluralité des intérêts de connaissance, les uns conduisant plutôt à étudier les contextes (et, précisément, tel ou tel type de contexte), les autres incitant au contraire à privilégier l'examen des dispositions (et, le cas échéant, de tel ou tel type de disposition incorporée) : «Il est légitime d'étudier le monde social à des échelles variées pour comprendre des niveaux de réalité sociale différents (le monde, le champ ou le système, le groupe, l'institution, l'organisation, l'interaction ou l'individu singulier) et d'analyser des aspects ou des dimensions variés des pratiques» (ibid.: 19). Mais encore convient-il de ne pas perdre en cours de route l'un des deux éléments (les «Dispositions» ou le «Contexte») de la formule scientifique censée rendre raison des «Pratiques» (et favoriser l'unification des sciences humaines et sociales).

Cette formule paraît surtout prometteuse («rentable scientifiquement» dit Lahire [ibid. : 22]) pour les recherches sociologiques focalisées sur la compréhension des singularités individuelles (au reste, on le sait, elle est directement issue de recherches de ce genre [2002, 2004]). C'est en travaillant à une échelle individuelle que l'on peut le mieux observer en pratique - dans les pratiques et dans les comportements - l'entrecroisement des dispositions incorporées et des contextes d'action. C'est à travers des entretiens approfondis qu'il est possible de se faire une idée des expériences « socialisatrices » à l'origine des dispositions et compétences qu'engage l'acteur dans des contextes spécifiques plus ou moins contraignants.

Tous les chercheurs n'en sont pas moins susceptibles de se mettre d'accord sur le fait que chacun est libre de privilégier l'échelle d'observation qui lui convient (l’idéal étant cependant de multiplier le plus possible les angles de vue). De même, estime Lahire, ils pourraient parfaitement s’interdire de concert de généraliser au-delà de ce qu'autoriseraient la contextualisation opérée 
ainsi que la méthodologie mise en œuvre. Le sociologue exhorte ainsi ses pairs à s'efforcer d'adapter scrupuleusement leurs opérations de contextualisation aux objets étudiés et à s'abstenir de toute généralisation abusive.

Il suffirait que ces conditions soient respectées pour que tout un chacun ait le sentiment réconfortant d'apporter sa pierre à l'édifice des sciences humaines et sociales, c'est-à-dire de contribuer à la réalisation d'un seul et même programme scientifique d'étude des comportements humains: "Souvent, les chercheurs ne travaillent que sur des aspects ou des parties d'un programme plus général consistant à rendre raison des pratiques sociales (de l'action, des comportements, etc.) à partir de la double prise en compte des contextes d'action et du passé incorporé des acteurs » (Lahire, 2012a : 320). Parce qu'ils sauraient déterminer lucidement, en fonction du type d'objet étudié et de leurs propres intérêts de connaissance, si la structuration du contexte d'action compte plus, pour eux, que l'investigation du passé incorporé des acteurs, ou apprécier correctement le rapport entre les méthodologies mises en œuvre et les échelles d'observation de la réalité sociale ou les types de contexte privilégiés par eux-mêmes et par les collègues de leurs disciplines et des autres disciplines, parce qu'ils sauraient, en somme, contextualiser à propos (et évaluer comme par réflexe, chez autrui, la pertinence de l'opération de contextualisation au regard des objets traités), les chercheurs, veut croire Lahire, œuvreraient collectivement à l'unification des sciences humaines et sociales : «Prendre conscience de la variation des échelles d'observation utilisées, des niveaux de réalité sociale visés et des types de faits étudiés, c'est, paradoxalement, gagner en largeur de vue et ressaisir l'unité des sciences humaines et sociales que la diversité des travaux finit par masquer aux yeux mêmes de ceux qui les produisent» (ibid. : 227). Tel est l'idéal qui gouverne sa lecture de très nombreux travaux de sociologie, d'histoire et d'anthropologie, et rend toujours stimulantes, en particulier, ses analyses critiques des principaux paradigmes sociologiques (de l'interactionnisme symbolique à la théorie des systèmes sociaux de Luhmann en passant bien entendu par la théorie des champs). 


\section{Un raisonnement dichotomique}

Monde pluriel, on l'aura compris, est un livre de refondation théorique dense et maitrisé. De bout en bout, Lahire développe une argumentation claire et cohérente sur un plan conceptuel. Il facilite ainsi considérablement le travail de lecture critique. En somme, il s'expose d'autant plus à la critique qu'il donne beaucoup à penser.

De prime abord, ses propositions ne soulèvent aucune difficulté majeure. À y regarder de plus près, toutefois, sa manière de procéder par grandes dichotomies est souvent discutable (Atkinson 2012 : 170). Paradoxalement, et c'est ce qui fait toute son originalité, Lahire ne cesse de raisonner abstraitement sur un mode dichotomisant (l'extérieur est opposé à l'intérieur, le contexte aux dispositions, etc.) pour mieux défendre une théorie de la socialisation niant, ou évacuant, ce que beaucoup d'auteurs ont perçu comme une tension dichotomique réelle - la tension entre le «psychique » (lequel ne possède à proprement parler aucun statut dans sa sociologie, alors même - on y reviendra - qu'il s'agit de l'objet principal qu'il lui a assigné) et le « social ».

L'un des aspects peut-être les plus contestables de sa théorisation, à cet égard, réside dans le caractère flou de la distinction entre contextes «socialisateurs » et contextes d'activation ou de mise à l'épreuve des «expériences socialisatrices». Distinction qui devrait recouper, en bonne logique, du moins en partie, la distinction implicite entre «contextes passés» et «contextes présents ». Lahire écrit notamment :

Le contexte présent de l'action peut être considéré de deux points de vue différents : en tant que cadre déclencheur de dispositions incorporées, ou bien en tant que cadre socialisateur des acteurs. Lorsque les acteurs en question sont des enfants, on voit immédiatement dans quelle mesure les contextes d'action sont aussi des contextes de socialisation, donc des cadres où se forment des compétences, des appétences ou des habitudes mentales et comportementales. [...] Mais même lorsqu'il s'agit d'adolescents ou d'adultes, la fréquentation répétée de certains contextes d'action n'est jamais sans conséquences socialisatrices (ou, dit autrement, dispositionnelles) sur eux (2012a : 26). 
Sa façon d'évoquer la spécificité socialisatrice des «contextes d'action» du point de vue des enfants - avant de la relativiser - montre que son argumentation, ici, n’est pas des plus assurées. C'est assez rare chez lui pour être souligné. Comment discriminer significativement la manière dont un «contexte d'action» s'imposerait comme cadre socialisateur du mode selon lequel il opérerait en tant que cadre déclencheur de dispositions intériorisée ? Est-ce une question d'âge ? De types de contextes? La dichotomie «dispositions»/«contexte», on le voit, risque de produire des équivoques assez lourdes ${ }^{\mathrm{ii}}$. On ne comprend pas très bien comment ce qui « reste » - en tant que dispositions incorporées - des contextes socialisateurs passés s'articule avec ce qui se joue dans tel «contexte» présent; et comment ce qui se joue dans tel «contexte » présent à partir de ce «reste» est de nature à faire évoluer celui-ci ou à le renforcer. Serait-il possible d'identifier des moments à partir desquels les « contextes » cesseraient d'exercer sur les acteurs de réels effets «socialisateurs», c'est-à-dire, par exemple, des moments à partir desquels toute nouvelle relation ne consisterait structurellement qu'à reproduire les relations affectives vécues jusqu'à la fin de l'adolescence ou à confirmer les diverses expériences relationnelles accumulées en vue d'accéder à une certaine position « sociale » ? La « socialisation » - processus d'acquisition de dispositions et de compétences à travers la fréquentation de différents « contextes d'action »serait-elle un processus sans fin? Un «contexte d'action » donné pourrait-il être au principe du déclenchement de certaines dispositions tout autant qu'il pourrait favoriser la formation de nouvelles dispositions - et cela, à tout âge de la vie ? La formule «Passé incorporé + Contexte d'action présent $=$ Pratique » et, surtout, sa version la plus sophistiquée, « Produits intériorisés de la fréquentation passée de contextes d'action + Contexte présent = Pratiques observables» (ibid. : p. 26), qui parait surtout devoir s'appliquer aux adultes, soulèvent, immanquablement, et interminablement, des questions de ce type.

Il n'est pas illégitime non plus de se demander si les «contextes», du fait même de leurs spécificités dans le cadre des sociétés hautement différenciées et intégrées, ne seraient pas de 
nature à introduire des modifications dans la structure de la personnalité individuelle durant l'âge adulte sans commune mesure avec ce qu'il en aurait été dans les sociétés moins complexes ou avec ce que l'on pensait savoir de la faiblesse relative de la malléabilité du psychisme une fois passé le stade de l'adolescence. Le « contexte » est-il plus imprévisible dans les sociétés fortement différenciées que dans les sociétés faiblement différenciées, obligeant ainsi les acteurs à arbitrer consciemment entre des dispositions hétérogènes ? Qu'est-ce qui, de manière propre aux sociétés différenciées organisées en États, conférerait aux «contextes» un pouvoir socialisateur plus ondoyant et diffus, et aux modalités d'activation des «dispositions » une prédictibilité et une systématicité moins grandes (du fait de la nécessité, pour les individus, de gérer un patrimoine de dispositions éventuellement contradictoires) ? De même, on eût aimé être un peu plus renseigné quant à la façon dont il faudrait s’y prendre pour évaluer avec quelque exactitude le poids du "passé incorporé » des acteurs sur telle ou telle «scène d'action ». Dernière question que l'on serait enfin enclin à poser à Bernard Lahire, plus directement inspirée par des considérations psychanalytiques : dans quelle mesure les relations de plus en plus précoces du jeune enfant avec des personnes prenant en charge certaines tâches domestiques et d'éducation (comme l'employée de crèche, la nourrice ou la baby-sitter), qu'il invoque pour illustrer sa thèse d'une pluralisation récente des contextes de socialisation et d'une complexification subséquente de la constitution des patrimoines dispositionnels, viennent-elles fondamentalement modifier ce qui se cristallise dans la configuration triangulaire mère-père-enfant en termes de transmission de l'inconscient sexuel (Laplanche : 2007) ou du point de vue existentiel du rapport aux ascendants (Ortigues, Ortigues : 1984) $)^{\mathrm{iii}}$ ?

Le problème est sans doute que la théorie de la socialisation défendue par Lahire («contextes de socialisation pluriels $=$ dispositions hétérogènes ») n'est faite ni pour penser le processus de cristallisation d'une strate fondatrice de la structure de la personnalité individuelle sur la base des relations enfants-parents, ni pour expliquer la structuration d'ensemble de la société. Elle 
s’oppose délibérément, semble-t-il, à toute idée de hiérarchisation réalité-congruente des facteurs explicatifs des modes de configuration du «psychique » et du « social».

Ainsi, critiquant Bourdieu parce qu'il n'aurait pas tenu compte « des effets socialisateurs de la pluralité des cadres sociaux» (2012a: 140), Lahire tend-il à perdre de vue que la théorie de l'habitus, en dépit de ses limites (comme il l'a mis en valeur avec talent [2001]), présente au moins l'avantage d'attirer l'attention sur une sorte de tension structurale - manifestement à l'œuvre dans les «situations contemporaines»(2012a: 141) - entre la configuration de la famille (où se perpétue une logique mono-socialisatrice et de loyauté exclusive) et la configuration différenciée de ce que l'on pourrait appeler une société de «champs». Et il congédie peut-être trop rapidement une hypothèse qui eût mérité un minimum de considération, à savoir l’hypothèse bourdieusienne autant que freudienne, au fond - selon laquelle l'individu, en tant que «produit» à bien des égards «mono-socialisé » (dans sa famille), ne découvrirait « la pluralité extérieure à lui que dans un second temps » (ibid. : 140).

On peut pourtant difficilement donner tort à Lahire lorsqu'il reproche à Bourdieu de penser la famille comme un «champ ». Sauf que cette erreur - pointée à juste titre - est peut-être du même ordre que celle qui consiste à aborder la famille comme un cadre de socialisation simplement distinct des autres cadres de socialisation par quelques traits propres qui ne se comprendraient que par rapport aux traits généraux des «contextes d'action» composant le monde social. En substance, Lahire nous dit que la famille constitue un univers primaire (celui à partir duquel l'enfant est introduit au monde) et non spécialisé. Mais il est ambivalent. D’un côté, ignorant totalement la sexualité infantile, il note que «la socialisation familiale [...] va constituer les dispositions mentales et comportementales au principe des engagements et des relations contractées par la suite» (ibid.: 175). D’un autre côté, il répertorie et sur-interprète toutes les entailles supposées au monopole primo-socialisateur de la famille ; cela, consciemment ou non, afin d'asseoir un schéma théorique général «niveleur» mettant en corrélation cadres pluriels de socialisation et patrimoines de dispositions plurielles (voire, dans certains cas, hétérogènes et 
franchement contradictoires). Or, manifestement, l’idée selon laquelle la famille, certes distincte du «champ », constitue un « contexte » de socialisation parmi d'autres s'oppose assez nettement à la perspective freudo-bourdieusienne postulant, sur des bases empiriques solides (que Lahire luimême valide partiellement), que les primo-expériences relationnelles intrafamiliales ont un effet structurant au sens où elles déterminent à la fois l'occurrence et la réception des expériences ultérieures (ce qui pose entre autres la question de la place et de la permanence de la sexualité dans l'existence humaine).

Articuler psychanalyse et sociologie des champs

Lahire a ainsi un certain mal à se situer par rapport à la sociologie des champs et à la psychanalyse. Ces deux grands cadres de pensée reflètent par excellence, par ce qui les rend à la fois si proches l'un de l'autre et si étrangers l'un à l'autre, la tension historiquement constituée entre la configuration familiale (érigée en sphère d'intimité et d'accomplissement affectif possédant en elle-même sa propre fin) et la configuration de l'espace social dans son ensemble (fortement différencié et structuré par différents sous-espaces sociaux tous liés d'une façon ou d'une autre à l'État en tant que méta-champ du pouvoir). Tension que le système scolaire et universitaire - espace intermédiaire, filtre incontournable - se charge plus ou moins bien de résorber et qu'il traduit sans cesse, coincé qu'il est entre les besoins spécifiques de ces deux configurations relationnelles. Tension dont on peut dire qu'elle caractérise les sociétés ayant succédé à la «dernière grande formation non bourgeoise de l'Occident» (Elias 1985: 15), à savoir la société de cour (société structurée par la cour).

Ce fait objectif non seulement pourrait permettre de guider avec quelque sûreté l'appréhension immédiate de la «psyché » et de la « société », mais aider à ordonner les facteurs explicatifs de ces deux ordres de réalité ainsi que les diverses théories des sciences humaines et sociales. 
L'épistémologie lahirienne, toutefois, n'en tient pas vraiment compte. Certes, rien n'est moins simple que de réfléchir à la manière d'articuler deux théories visant toutes deux à promouvoir une sorte de hiérarchisation réalité-congruente des principes explicatifs du comportement humain, chacune pour son «ordre» propre, la structuration différenciée globale de la société et ses conséquences sur la structure de la personnalité à travers l'« habitus », pour l'une, les racines du fonctionnement conflictuel intrapsychique par entrechoquement des ressorts pulsionnels du processus de maturation humaine et de la relation triangulaire fantasmatique père-mère-enfant, pour l'autre (Joly 2012 : 61-62, 129). Contournant la difficulté, Lahire contourne aussi nombre de faux-problèmes et apporte des clarifications et précisions extrêmement utiles (on pense par exemple à la distinction conceptuelle lumineuse qu'il opère entre "autonomie-spécificité » et « autonomie-indépendance » [2012a : 77-106]). Mais il n'est pas sans s'enfermer, en même temps, dans une posture épistémologique qui trahit certains points aveugles de sa théorie de la socialisation.

Non pas que sa principale proposition, à savoir que nous sommes multi-déterminés, soit en tant que telle infondée. Les êtres humains des sociétés différenciées, dit-il, sont amenés à fréquenter plus ou moins précocement, durablement et systématiquement divers «contextes d'action » qui sont aussi des cadres socialisateurs (famille, crèche, école, entreprise, club sportif, groupe de pairs, etc.) (ibid.: p. 25-26). De là, la formation complexe et jamais achevée une fois pour toute de patrimoines de dispositions et de compétences hétérogènes et potentiellement contradictoires, qui peuvent être activées ou non en fonction des situations telles qu'elles se présentent une à une. Il est permis de douter, cela dit, que les primo-relations du nourrisson puissent rigoureusement être décrites en termes de «fréquentation» de quelque «contexte d'action» familial. C'est pourtant ce qu'implique le schéma théorique «dispositionnalistecontextualiste » de Lahire. Il se garde certes de condenser sa théorie de la socialisation dans les sociétés différenciées dans une formule d'une simplicité comparable à celle par laquelle il ne cesse de résumer sa théorie de la pratique: «Dispositions + Contexte = Pratique ». La formule 
fantôme « Monde pluriel $=$ Homme pluriel » n'en plane pas moins en permanence au-dessus de ses démonstrations. Cela confère à son livre une grande densité, car il sait jongler habilement avec ces différents registres théoriques. Mais, à notre avis, cela pose aussi nombre de problèmes laissés irrésolus.

Le champ, un « contexte » parmi d'autres?

C'est dans sa façon d'aborder la théorie des «champs » et la réalité des «champs » - et de se positionner par rapport à Bourdieu - que Lahire se montre le plus radical et prend par conséquent le plus de risques.

Il est bien sûr tout à fait libre de confronter systématiquement la notion de « champ » à celle de «monde» et de les mettre pour ainsi dire sur un pied d'égalité (en tant par exemple que « théories régionales à prétention générale [...] empiriquement plus productives » que les théories de Parsons et de Luhmann sur les systèmes sociaux [ibid. : 60]). Mais il nous semble que ce n'est possible qu'à la faveur d'une double réduction, le «champ » étant considéré à la fois comme un type de microcosme parmi beaucoup d'autres au sein des sociétés étatiques fortement différenciées et intégrées, et comme une opération de contextualisation déterminée par un intérêt de connaissance précisément circonscrit (qui porterait à titre exclusif sur les luttes entre dominants pour l'appropriation ou la redéfinition d'un capital spécifique, c'est-à-dire sur une seule des logiques régulatrices - différente par exemple de la logique «coopérative » mise en exergue par la théorie des «mondes » - du sous-espace considéré).

De même, Lahire va jusqu’à dénier à Bourdieu le droit de critiquer Goffman sur un plan général :

Il existera toujours des chercheurs pour reprocher à E. Goffman, à partir d'une opération de contextualisation différente, d'autonomiser les échanges linguistiques. C'est ce que fait, entre autres, 
P. Bourdieu en soutenant que la vérité de l'interaction n'est pas tout entière contenue dans l'interaction. Le cadre immédiat de l'interaction ne serait pas le contexte approprié. Pris par une sorte de réalisme de la structure, convaincu que son point de vue est le seul possible et qu'il n'existe donc pas plusieurs intérêts de connaissance, il ne peut voir dans les démarches interactionnistes que leur aspect fautif (ibid. : 255-256).

On peut admettre avec Lahire que l'intérêt porté par Bourdieu à l'interaction vendeur-acheteur (effective par exemple lors d'un Salon de la maison individuelle) n'est pas du même ordre que celui porté par les « ethnométhodologues conversationnalistes ou par les interactionnistes » (ibid. : 254). Mais, ici, la question est de savoir si l'approche bourdieusienne ne se distinguerait pas en donnant les moyens de comprendre, en même temps, ce qui se joue bic et nunc dans une interaction et la manière dont la société est structurée dans son ensemble (en sorte par exemple que le vendeur, dans l'interaction en cause, apparait contre toute attente sous les traits d'un agent d'État qui s'ignore [Bourdieu 2012: 34]). Si tel est le cas, alors il n'y a rien d'embarrassant à juger que raisonner en termes de «champ» est préférable, dans l'absolu, à l'opération de contextualisation réalisée par les interactionnistes. Tout n'est donc pas seulement une question d'angle de vue. Il y a des angles de vue qui offrent la possibilité de saisir comment la société dans son ensemble est structurée. D'autres qui ne l'offrent pas (même s'ils peuvent nous renseigner - à l'instar de l'interactionnisme symbolique - sur la personnalité et les comportements des individus de cette société). Le «champ» n'est pas un contexte parmi d'autres; c'est un espace de relations objectives qui structure un espace plus large en interaction conflictuelle avec d'autres espaces isomorphes tous infiltrés d'une certaine manière par l'État (ou, aurait dit Bourdieu, par la pensée d'État). Les sociétés de «champs» sont des sociétés marquées par une forte différenciation fonctionnelle et puissamment intégrées grâce au développement des institutions étatiques. Elles sont structurées par un ensemble d'espaces qui se présentent - de manière plus ou moins crédible et affirmée, sur le modèle « fondateur » de l'espace économique - comme des sphères autonomes dont les principes sont censés valoir pour la société tout entière et suffire par eux-mêmes à 
orienter l'évolution sociale, mais qui, en réalité, sont fonctionnellement interdépendants et reliés aux structures étatiques.

Ainsi, parce qu'elle rend pensable la répercussion des structures sociales et politiques les plus objectives sur les interactions les plus fines, la théorie des «champs » est la seule de toutes les théories sociologiques examinées par Lahire qui soit de nature à fournir des éléments de réponse tangibles à la question de savoir ce qui fait tenir ensemble les sociétés différenciées. Étant entendu que le fait d'établir que les sociétés actuelles sont structurées par les «champs » et par l’État en tant que méta-« champ », et qu’il y a là une caractéristique historique récente du mode de structuration globale du «social», ne signifie pas qu'il ne soit pas possible de repérer dans les sociétés antérieures des linéaments des « champs ». Grâce à cette idée de structuration, toutefois, on s'épargne la peine de déterminer depuis quand les « champs » existent.

\section{La théorie des « champs » comme « théorie hiérarchique »}

La lecture de Monde pluriel n'est pas sans procurer une sensation de vertige analogue à celle dont parlait récemment Sabina Loriga à propos de la micro-histoire (Loriga 2010 : 162). On se demande en effet où s'arrête et ce qui structure le travail de repérage et de classification des types de dispositions et des types de contextes d'action qu'appelle à entreprendre Bernard Lahire. S'agissant des «champs", on dirait qu'il voudrait pouvoir les identifier une fois pour toutes, en distinguant leurs propriétés générales de celles des autres types de microcosmes et en spécifiant les propriétés de chacun pris isolément; idéalement, il aimerait pouvoir les dénombrer avec précision. Le souci de rigueur scientifique dont il témoigne est admirable. Mais l'on pourrait lui objecter que les frontières des « champs » sont toujours mouvantes, et que ces espaces ne cessent de projeter vers l'extérieur les valeurs et représentations qui s'y définissent à travers les luttes de leurs agents - et contribuent concurremment, par là, à structurer le vivre-ensemble de l'espace 
social tout entier (en même temps qu'ils font reconnaître la légitimité des lois qui les régissent). C'est pourquoi on ne peut ni les isoler totalement les uns des autres, ni les classer avec la méticulosité du taxinomiste. C'est pourquoi, aussi, leur analyse constitue un préalable indispensable à la compréhension des relations humaines dans leur ensemble à l'intérieur des sociétés étatisées-différenciées. C'est pourquoi, enfin, Bourdieu n’a jamais cherché à les enfermer dans une définition claire, nette et précise.

Lahire prête à ce dernier l'idée selon laquelle «les champs couvrent l'ensemble des microcosmes possibles dans l'espace social»(2012a: 158). Mais il eût été tout aussi légitime d'écrire que les champs, pour Bourdieu, sont les microcosmes qui, du fait de leurs caractéristiques principielles (luttes symboliques d'autonomie, rapports dominants/dominés, répartition hiérarchique et perception relationnelle des capitaux, etc.), ont pour effet de structurer à des degrés divers et concurrentiellement l'ensemble de l'espace social, et pour fonction de canaliser la libido de tous les membres de cet espace (qui, pour la majorité, ne participent qu'indirectement ou par procuration aux luttes qui se déroulent au sein des «champs»). C'eût été privilégier la visée générale et l'intention profonde de la théorie des «champs ». Car il est frappant qu'à aucun moment Lahire n'envisage cette théorie comme une théorie biérarchique (dans l'esprit de ce que S. J. Gould a fait de la théorie darwinienne et de ses conceptions d'historien des sciences [2006]) et les réalités qu'elle prétend interpréter comme des réalités structurantes (les «champs» en tant que tels) et comme des réalités structurant une réalité structurée (l'espace social tout entier). Or la société globale n'a pour axe de structuration le volume et la structure du capital culturel et du capital économique possédés, comme le reconnaît Lahire (2012a: 163), que parce qu'elle est une société structurée par les «champs » et par l'État comme méta-«champ » parvenant, lui et lui seul, à revendiquer avec succès le monopole de la définition légitime de l’Universel.

Mais ce qui est exclu par la porte finit toujours par revenir par la fenêtre, et c'est dans un addenda (ibid. : 353-363) que, nous livrant sa théorie générale de la différenciation sociale sous la forme de schémas, Lahire suggère que les «champs» pourraient bien avoir des propriétés 
structurantes du point de vue de l'espace social global. Il se représente en effet la société comme constituée de différents compartiments ou domaines d'activité séparés, tous soumis à la même stratification entre les trois grandes classes sociales (supérieures, moyennes, populaires). Toutefois, on aurait aimé mieux comprendre comment les différents compartiments sont reliés les uns aux autres et, surtout, comment l'activité des sommets - qui se trouvent être des « champs » et sont implicitement revêtus d'une fonction directrice - se répercute dans les étages inférieurs.

\section{Le sociologue face aux sciences du psychisme}

La psychanalyse, en tant que théorie hiérarchique mettant l'accent sur des effets structurants (dans le processus de fabrication concaténationnelle du psychisme), est tenue différemment en lisières que la théorie des champs. On trouve sous la plume de Lahire - qui s’intéresse essentiellement à la pensée freudienne sous l'angle des relations intrafamiliales précoces - beaucoup d'idées ingénieuses et originales. Il est discutable, cela dit, que poser «la question de l'échelle d'observation et du niveau de réalité sociale visé » (ibid. : 264-279) suffise à rendre la psychanalyse compatible avec les sciences sociales - ne serait-ce que parce que le problème de l'inconscient sexuel ou de la névrose infantile poursuit les êtres humains toute leur vie, imprégnant l'ensemble de leurs relations, outre qu'il est difficilement interprétable en termes de constitution de «dispositions». Quoi qu'il en soit, la psychanalyse, d'un point de vue sociologique « dispositionnaliste » (que les psychologues gagneraient beaucoup à ne pas ignorer), aurait le tort de se concentrer exclusivement sur les interactions intrafamiliales et de donner la priorité à quelques interactions libidinalement signifiantes de la prime enfance.

De la psychanalyse, Lahire retient aussi l’idée de pluralité/conflictualité du psychisme (qu'elle a été la première à mettre en évidence sur un mode systématique). Le sujet est soumis, en effet, à 
des exigences internes contraires. Mais, pour le sociologue, les conflits internes ne sont que le reflet de conflits externes (entre les parents, dont les injonctions se contrediraient, ou entre les parents et les membres de telle ou telle institution d'éducation ou de soins, dont les conceptions divergeraient, etc.). Les conflits intrapsychiques, à le suivre, seraient l'une des manifestations spécifiques des sociétés "plurielles» hautement différenciées. On retrouve sa théorie de la socialisation («Monde pluriel» $=$ «Homme pluriel») et son style argumentatif dichotomisant. Les psychologues ont pourtant montré que la relation, pour le jeune enfant, est par nature source de béatitudes autant que de frustrations intolérables ; autrement dit, que le conflit intrapsychique est originaire et structurel-structurant. Tout au long de la vie être en relation avec autrui pose problème tout en répondant à des impératifs biologiques incontournables. Mais Lahire récuse avec force l'idée que le fonctionnement intrapsychique conflictuel puisse traduire une sorte de heurt inévitable entre des besoins internes innés de satisfaction pulsionnelle et les contraintes de la vie en société (qui imposent notamment d'acquérir les schémas d'autorégulation pulsionnelle et de comportement culturellement établis [Elias 2010]). Cet aspect de l'humanité - qui reste à éclaircir de manière adéquate - est totalement exclu de son horizon de pensée. Ainsi Freud a-t-il beau s'efforcer de marcher tant bien que mal sur deux jambes - le «biologique » et le «socialfamilial » - et de penser la genèse du psychisme à l'intersection des pulsions innées et des primorelations parents-enfants, Lahire lui enjoint de sauter à cloche-pied : «Il faut résolument prendre le parti de Freud sensible à l'“influence parentale" [...] contre celui de Freud [...] invoquant les “prédispositions héréditaires" ou “innées" " (2012a : 128). Mais pourquoi faudrait-il choisir à tout prix ?

Lahire donne bien souvent le sentiment que le sociologue doit refaire intégralement le chemin parcouru avant lui par le psychologue ou par le psychanalyste. Il écrit ainsi que «le chercheur» (il faut comprendre : le sociologue) doit, pour cerner par exemple « la nature des liens d'amour ou d'amitié que nouent telle ou telle personne, [...] se mettre en quête du même genre de passé auquel s'attache la psychanalyse freudienne (fond d'expériences familiales remontant à la petite 
enfance) » (ibid. : 227). Il ne semble pas considérer que le sociologue puisse - doive - faire usage des concepts de la psychanalyse ou faire fructifier les acquis de celle-ci. Comme s'il fallait tout reprendre à zéro, comme s'il n’y avait pas d'autre possibilité que de partir d'une table rase (ce qui n'est pas sans consonner, justement, avec cette théorie officielle des sciences sociales qu'est la théorie de la Table rase dénoncée avec verve par Steven Pinker [2005] $]^{\text {iv }}$.

Le fait est qu'il s'adresse essentiellement aux sociologues. C'est la sociologie qu'il veut transformer en la faisant rentrer dans les familles et en la réorientant vers l'examen approfondi des singularités individuelles et des « cas improbables ». C’est à partir de cette nouvelle sociologie qu'il projette d'unifier l'ensemble des sciences humaines et sociales (sans que l'on sache très bien quelle place est réservée à la psychologie dans cette perspective). Il n'empêche : s'il s'est placé sur un terrain relativement vierge du point de vue de sa discipline, en proposant ni plus ni moins que de jeter les fondements d'une sociologie ayant pour objet la genèse du psychisme (une sociologie psychogénétique, car l'on ne voit pas comment l'étude des patrimoines de dispositions et de compétences pourrait éviter d'être en même temps une étude de la structuration du psychisme) $)^{\mathrm{v}}$, ce terrain est balisé depuis bien longtemps par diverses approches psychologiques, de la thérapie familiale systémique à la psychanalyse en passant par la psychologie du développement. Les évolutions de la famille ou les «souffrances familiales » ont par exemple été éclairées récemment par des psychologues, des psychiatres ou des psychothérapeutes, à partir d'expériences cliniques «familiales» (ou « relationnelles ») extraordinairement riches (Andolfi et al. 1985; Angel, Mazet 2004). Mais Lahire, lui, non sans courage, a préféré se lancer seul dans l'entreprise consistant à renouveler le vocabulaire de la sociologie sur la base d'études quasi ethnographiques de la famille et d'enquêtes par entretiens censées rendre raison des patrimoines dispositionnels des individus saisis un par un $(1995,2002,2004)$.

Dans l'idéal, la réorientation des concepts de la sociologie envisagée par Lahire devrait aller de pair avec une réorientation des concepts de la psychologie; le tout, dans un même esprit processuel dé-réifiant ${ }^{\mathrm{vi}}$. Force est de constater qu'on est actuellement loin du compte et que les 
cloisons entre disciplines demeurent étonnamment étanches. Il se peut que Lahire soit gêné par la tendance de certains théoriciens de la thérapie familiale à opposer la dynamique intrinsèque des constellations familiales aux nécessités de la «socialisation» (entendue en un sens restreint comme ce qui prépare à la vie en société) (Hefez 2004 : 13). Poser le problème n'eût pourtant pas été inutile, alors que la notion large de «socialisation », pour Lahire, semble autant aller de soi que la réalité qu'elle recouvre. De même, il eût été intéressant de situer la sociologie « dispositionnaliste » par rapport à la succession des stades du développement psychologique de l'enfant distingués par Henri Wallon (qui, du reste, se superposent aux stades freudiens du développement libidinal infantile) (Jalley 1981) $)^{\mathrm{vii}}$. Au risque de se répéter, une sociologie des dispositions - qui ne peut pas ne pas être une sociologie de la genèse du psychisme - a des comptes à rendre aux approches psychologiques, la psychanalyse en particulier, qui ont su donner à la notion de «psychisme» un réel contenu en le rapportant tout à la fois au processus de maturation biologique spécifique à l'être humain et à la configuration de relations humaines (la configuration père-mère-enfant dite « edipienne» par les psychanalystes) structurant dans ses premières phases un tel processus sous la forme de l'inconscient.

Quant à saisir « les pratiques au croisement des propriétés sociales des acteurs et des propriétés sociales des contextes» (Lahire 2012a : 21), c'est là un objectif que la psychologie sociale s'est donné de longue date, peut-être la première, à la faveur il est vrai de l'application de méthodes expérimentales (mais il eût été instructif, justement, que Lahire se confrontât aux expériences de psychologie sociale ayant mis en évidence la faible résistance ou opérabilité des dispositions et/ou valeurs intériorisées face à certains effets de contexte). Ainsi, les thèmes de la dissonance cognitive (Vaidis 2011) et de l'interaction facteurs dispositionnels (personnalité)/facteurs contextuels (situation) sont parmi les plus privilégiés par la psychologie sociale sans que le sociologue n'ait un seul mot pour cette discipline.

Il prête donc le flanc à la critique en faisant l'éloge de l'interdisciplinarité tout en mettant à distance les disciplines ou sous-disciplines les plus proches, objectivement, de sa manière de faire 
de la sociologie. Le problème provient sans doute de la spécificité de sa position et de ses prises de position, puisqu'il se trouve de facto en situation de pouvoir plaider en tant que sociologue et au nom de la sociologie pour l'unité des sciences sociales, c'est-à-dire de renouer avec une ambition durkheimienne, alors même qu'il a fait le pari de l'individuel et du singulier au détriment de l'étude exclusive des faits et objets sociaux massifs sur lesquels la sociologie dite « scientifique » a fondé historiquement sa légitimité, avec force statistiques à l'appui.

\section{Bonheur de la création intellectuelle}

Mais ce n'est pas assez de dire que Bernard Lahire, dans Monde pluriel, innove sur un plan théorique et excelle dans l'art de la clarification conceptuelle synthétique. Il a lu tous les auteurs importants de sa discipline, de très nombreux travaux d'histoire, d'anthropologie, de linguistique, de psychanalyse, etc. C'est également un fin connaisseur des œuvres philosophiques de Hume et Wittgenstein (même si l'on peut se demander si l'usage qu'il en fait aide vraiment à relever le défi scientifique d'une réorientation processuelle réalité-congruente des instruments de pensée afin de rendre compte le plus précisément possible des relations humaines sous tous leurs aspects).

Il était par conséquent l'un des mieux placés pour analyser les causes et dénoncer les conséquences délétères de la spécialisation scientifique excessive que nous subissons depuis un certain nombre d'années. Il livre, en conclusion de son essai, une critique jubilatoire des normes actuelles d'évaluation et invite les chercheurs à militer «pour que soit ralenti le rythme des recherches, comme celui des publications, et allongé le temps consacré à leur "évaluation" » (2012a: 349). Il ne le dit pas explicitement, mais on sent qu'il pense que la plupart des chercheurs, aujourd'hui, ne sont pas épanouis : ils sont soumis à des procédures d'évaluation dont le caractère absurdement bureaucratique les écœure ; ils sont conduits insidieusement à privilégier la quantité à la qualité ; leurs travaux ne sont lus - au mieux - que par une poignée de collègues- 
spécialistes ayant tous et toujours, comme eux, le nez dans le guidon; ils n'ont plus guère de temps pour la lecture "gratuite» et «désintéressée », etc. On pourra lui reprocher de ne pas apporter de solutions concrètes pour remédier à cet état de fait. Mais le plus important est le témoignage qu'il nous apporte par son œuvre, de ses Tableaux de famille (1995) de jeune sociologue exceptionnellement doué à son magistral Franz Kafka (2010), à savoir qu'il y a un vrai bonheur du travail intellectuel lorsqu'il peut s'accomplir librement, de manière à la fois créative et rigoureuse. 


\section{Ouvrages cités}

Andolfi, Maurizio et al. 1985. La forteresse familiale : un modèle de clinique relationnelle, Paris, Dunod.

Angel, Pierre, Philippe Mazet, 2004. Guérir les souffrances familiales, Paris, PUF.

Atkinson, Will. 2012. «Where Now for Bourdieu-inspired Sociology ?», Sociology, vol. 46, n 1 : 167-173.

Bourdieu, Pierre. 2012. Sur L'État. Cours au Collège de France 1989-1992, Paris, Raisons d'agir/Seuil.

Dejours, Chistophe. 2009. Les dissidences du corps. Répression et subversion en psychosomatique, Paris, Payot \& Rivages (Petite bibliothèque Payot).

- 2013 [2009]. Travail vivant. 1. Sexualité et travail, Paris, Payot \& Rivages (Petite bibliothèque Payot).

Elias, Norbert, 1985 [1969]. - La société de cour, Paris, Flammarion (éd. orig., Die böfische Gesellschaft, Neuwied, Luchterhand).

- 2010. Au-delà de Frend. Sociologie, psychologie, psychanalyse, Paris, La Découverte (Textes à l’appui/Laboratoire des sciences sociales).

Essertier, Daniel. 1927. Psychologie et sociologie. Essai de bibliographie critique, Paris, Félix Alcan (Publications du Centre de documentation sociale).

Gould, Stephen Jay. 2006 [2002]. La structure de la théorie de l'évolution, Paris, Gallimard (Nrf Essais), (éd. orig., The Structure of Evolutionary Theory, Cambridge, Belknap Press of Havard University Press).

Grossetti, Michel. 2012. «Au-delà des champs », lectures.revues.org/8751.

Hefez, Serge. 2004. Quand la famille s'emmêle, Paris, Hachette Littératures .

Jalley, Émile. 1981. Wallon lecteur de Freud et Piaget, Paris, Éditions sociales.

Joly, Marc, 2012. Devenir Norbert Elias. Histoire croisée d'un processus de reconnaissance scientifique : la réception française, Paris, Fayard (Histoire de la pensée).

Klein, Melanie. 1968 [1947]. Essais de psychanalyse (1921-1945), Paris, Payot (Sciences de l'homme), (éd. orig., Contributions to Psychoanalysis : 1921-1945, Londres, Hogarth Press).

Lahire, Bernard. 1993. Culture écrite et inégalités scolaires. Sociologie de l'«échec scolaire »à l'école primaire, Lyon, PUL.

- 1998. L'bomme pluriel. Les ressorts de l'action, Paris, Nathan (Essais \& Recherches). 
- 2001 [1999]. «De la théorie de l'habitus à une sociologie psychologique», in Bernard Lahire (dir), Le travail sociologique de Pierre Bourdieu. Dettes et critiques, Paris, La Découverte/Poche (Sciences humaines et sociales): 121-152.

- 2002. Portraits sociologiques. Dispositions et variations individuelles, Paris, Nathan (Essais \& Recherches).

- 2004. La culture des individus. Dissonances culturelles et distinction de soi, Paris, La Découverte (Textes à l’appui/Laboratoire des sciences sociales).

- 2010. Franz Kafka. Éléments pour une théorie de la création littéraire, Paris, La Découverte (Textes à l’appui/Laboratoire des sciences sociales).

- 2012a. Monde pluriel. Penser l’unité des sciences sociales, Paris, Seuil (La couleur des idées).

-2012b [1995]. Tableaux de famille. Heurs et malheurs scolaires en milieux populaires, Paris, Seuil (Points-Essais).

Laplanche, Jean, 2007. Sexual. La sexualité élargie au sens freudien 2000-2006, Paris, PUF (Quadrige).

Loriga, Sabine. 2010. Le petit X. De la biographie à l'bistoire, Paris, Seuil (Librairie du XXI siècle).

Mahler M., Pine F., Bergman A., 1975. - The Psychological Birth of the Human Infant, New York, Basic Books.

Ortigues, Edmond, Marie-Cécile Ortigues. 1984. Edipe africain, Paris, L’Harmattan.

Rousseau-Dujardin, Jacqueline. 1986. «L'homme de la situation », Études freudiennes, nº 28 : 53-62.

Pinker, Steven. 2005 [2002]. Comprendre la nature humaine, Paris, Odile Jacob (éd. orig., The Blank Slate : The Modern Denial of Human Nature, New York, Viking).

Vaidis, D. C. F. 2011. La dissonance cognitive. Approches classiques et développements contemporains, Paris, Dunod (Sociale).

\footnotetext{
${ }^{\mathrm{i}}$ Monde pluriel a été assez peu discuté sur le fond. On relèvera une exception (Grossetti 2012). De manière générale, l'œuvre de Lahire, pourtant largement connue et diffusée, y compris à l'étranger, ne semble pas discutée comme elle mériterait de l'être.

ii « Contexte » est un concept qui symbolise par excellence ce qui est extérieur à l’être humain par opposition à ce qui lui est intérieur. Toute personne et toute institution, en ce sens, peuvent être considérées comme des «contextes ». La notion parait ainsi moins heuristique que celle de «disposition». Peut-être eutt-il été préférable de viser une construction conceptuelle permettant d'emblée de s'abstraire de la dichotomie « contexte »/« dispositions »? C'est ce
} 
qu'a tenté de faire Norbert Elias, et Lahire, c'est révélateur de sa façon de procéder, le range dans la catégorie des « contextualistes » oublieux du «passé incorporé » (2012a : 36).

iii Sur un plan théorique, nous semble-t-il, l'un des grands apports de la psychanalyse est de mettre l'accent sur le «réel» imposé par «l'antériorité des géniteurs» (Rousseau-Dujardin 1986: 61) ainsi que sur l'idée selon laquelle le jeune enfant ne peut pas ne pas produire des traductions imparfaites ou incomplètes des messages qui lui sont transmis par ses «parents» ou par les personnes qui lui en tiennent lieu - sans trancher ici la question de savoir jusqu’à quel point ces messages sont « compromis » par l'inconscient sexuel adulte (Dejours 2013 : 95-97).

iv En sorte que si Lahire esquisse un rapprochement des points de vue neuroscientifique et sociologique (2012a : 2627), c'est, dirait-on, pour mieux se tenir éloigné des acquis de la psychologie du développement, de la psychologie de l'évolution ou des différentes théories psychanalytiques ou psychologiques «stadistes » du développement affectif. v Il n’est d'ailleurs pas anodin que Lahire ait cessé de se réclamer d’une «sociologie psychologique » $(1998,2001)$.

vi Norbert Elias pourrait y aider, mais aussi un auteur comme Christophe Dejours à travers sa critique de la causalité psychosomatique et ses belles réflexions sur les « dissidences du corps» (2009).

vii Dans cette perspective de mise en corrélation des registres relationnels et des caractéristiques du processus humain de maturation physiologique et psychologique, même l'Edipe précoce de Melanie Klein, qui apparaitt chez l'enfant en tant qu'il ne comprend pas le langage, doit être considéré au moins sur un plan théorique (1968: 370-424). Quant au travail de Margaret Mahler et de ses élèves, il mériterait d'autant plus d'être pris en compte par le sociologue de la genèse du psychisme qu’il repose sur l'étude du «normal» (période symbiotique + séparation/individuation) pour étudier le "pathologique » (notamment la psychose) (Mahler, Pine, Bergman 1975), selon une « entrée » opposée à celle que Lahire reproche implicitement à la psychanalyse de privilégier. 